\title{
Atopic asthmatic patients have reduced airway inflammatory cell recruitment after inhaled endotoxin challenge compared with healthy volunteers
}

\author{
Michelle L. Hernandez, MDD,b, Margaret Herbst, MSN, RN ${ }^{a, b}$, John C. Lay, DVM, PhD ${ }^{a, b}$, Neil \\ E. Alexis, PhD a,b Willie June Brickey, $\mathrm{PhD}^{\mathrm{c}}$, Jenny P. Y. Ting, $\mathrm{PhD}^{\mathrm{c}}$, Haibo Zhou, $\mathrm{PhD}^{\mathrm{a}, \mathrm{d}}$, \\ and David B. Peden, MD, MS ${ }^{a, b, c}$ \\ ${ }^{a}$ Center for Environmental Medicine, Asthma and Lung Biology, University of North Carolina at \\ Chapel Hill School of Medicine \\ bepartment of Pediatrics, University of North Carolina at Chapel Hill School of Medicine \\ 'Department of Microbiology and Immunology, University of North Carolina at Chapel Hill School \\ of Medicine
}

${ }^{d}$ Department of Biostatistics, Gillings School of Global Public Health, University of North Carolina.

\begin{abstract}
Background-Atopic asthmatic patients are reported to be more sensitive to the effects of environmental endotoxin (LPS) than healthy volunteers (HVs). It is unknown whether this sensitivity is due to dysregulated inflammatory responses after LPS exposure in atopic asthmatic patients.
\end{abstract}

Objective-We sought to test the hypothesis that atopic asthmatic patients respond differentially to inhaled LPS challenge compared with HVs.

Methods-Thirteen allergic asthmatic (AA) patients and 18 nonallergic nonasthmatic subjects (healthy volunteers $[\mathrm{HVs}]$ ) underwent an inhalation challenge to 20,000 endotoxin units of Clinical Center Reference Endotoxin (LPS). Induced sputum and peripheral blood were obtained at baseline and 6 hours after inhaled LPS challenge. Sputum and blood samples were assayed for changes in inflammatory cell numbers and cytokine and cell-surface marker levels on monocytes and macrophages.

Results-The percentage of neutrophils in sputum (\%PMN) in induced sputum similarly and significantly increased in both HVs and AA patients after inhaled LPS challenge. However, the absolute numbers of leukocytes and PMNs recruited to the airways were significantly lower in AA patients compared with those seen in HVs with inhaled LPS challenge. Sputum levels of IL-6 and

\section{(C) 2012 American Academy of Allergy, Asthma \& Immunology}

Corresponding author: Michelle L. Hernandez, MD, Center for Environmental Medicine, Asthma and Lung Biology, 104 Mason Farm Rd, UNC School of Medicine, Chapel Hill, NC 27516. Michelle_Hernandez@med.unc.edu..

Disclosure of potential conflict of interest: M. L. Hernandez has received research grants and support for travel expenses from the National Institutes of Health (NIH)/National Institute of Allergy and Infectious Diseases (NIAID) and has received research grants from the National Institute of Environmental Health Sciences. M. Herbst has received grants from the NIH/NIAID. J. C. Lay has received research grants from the NIH. N. E. Alexis has received research grants from the NIH. W. J. Brickey has received research grants from the NIH and is employed by the NIH and the University of North Carolina. J. P. Y. Ting has received grants from the $\mathrm{NIH}$, has consulted for GlaxoSmithKline, has served on a National Institutes of Health Advisory Committee, and has received payment for the development of education presentations for the American Association of Immunologists. D. B. Peden has received grants from the US Environmental Protection Agency; the NIAID; the National Center for Research Resources; the National Heart, Lung, and Blood Institute; and the National Institute for Environmental Health Sciences. H. Zhou declares that he has no relevant conflicts of interest. 
TNF- $a$ were significantly increased in both cohorts, but levels of IL- $1 \beta$ and IL-18 were only significantly increased in the HV group. Cell-surface expression of Toll-like receptors 4 and 2 were significantly enhanced only in the HV group.

Conclusions-The airway inflammatory response to inhaled LPS challenge is blunted in AA patients compared with that seen in HVs and accompanied by reductions in airway neutrophilia and inflammasome-dependent cytokine production. These factors might contribute to increased susceptibility to airway microbial infection or colonization in AA patients.

\section{Keywords}

Asthma; LPS; induced sputum; inflammasome; innate immunity

The majority of asthma exacerbations are triggered by viral infections, pollutants that can injure or activate airway epithelial cells, or bioaerosols, which likely activate airway monocytes and macrophages. ${ }^{1-3}$ Endotoxin (LPS) is a pathogen-associated molecular pattern found in the outer layer of gram-negative bacteria and is an important component of ambient air particulate matter, ${ }^{4,5}$ tobacco smoke, and particulates in occupational and domestic environments. ${ }^{6,7}$

Inhaled LPS interacts with the cell-surface receptors CD14 and Toll-like receptor (TLR) 4 and activates airway macrophages, which in turn induce neutrophilic inflammation of the airways. In healthy volunteers (HVs) and allergic asthmatic (AA) patients, LPS inhalation has also been found to increase expression of CD11b and CD14 in airway monocytes and macrophages ${ }^{8-10}$ and increase levels of pro-IL-1 $\beta$ in airway macrophages. ${ }^{11}$ It has been reported that asthmatic patients are more sensitive to the effect of LPS exposure than HVs. ${ }^{9,12}$ We have observed that monocytes and macrophages from AA patients have increased expression of CD14 and that this factor correlates with airway eosinophilia and neutrophilic response to LPS, ${ }^{8}$ suggesting that asthmatic airway cells can be primed to respond to LPS challenge.

However, recent work has also shown that asthmatic patients might exhibit defective innate immune responses to microbes. Mycoplasma and Chlamydia species have been recovered from airway samples of both adult and pediatric asthmatic patients, ${ }^{13,14}$ thus making the use of macrolide antibiotics more popular for the treatment of asthma exacerbations and hard-tocontrol asthma. ${ }^{15}$ Large-scale epidemiologic studies have shown that asthmatic patients are at increased risk of airway infection with organisms such as Streptococcus pneumoniae ${ }^{16-19}$ compared with HVs. In addition, the airways of asthmatic patients have a different microbiome than those of healthy control subjects, with evidence of increased bacterial diversity ${ }^{20}$ and the presence of Haemophilus species ${ }^{21}$ in asthmatic patients.

It is presently unknown whether either the degree or character of response to microbial challenge is different between AA patients and HVs. In this study we undertook an initial test of the hypothesis that AA patients respond differentially to LPS versus HVs by comparing the airway inflammatory response of these 2 groups to inhalation challenge with a 20,000 endotoxin units (EU) dose of Clinical Center Reference Endotoxin (CCRE; LPS). Our primary end point was the percentage of neutrophils recovered from induced sputum after LPS inhalation. We undertook an exploratory analysis of end points focused on sputum neutrophil numbers, monocyte/macrophage surface marker expression, and sputum supernatant cytokine concentrations. We also examined changes in circulating leukocyte and blood neutrophil numbers after LPS challenge. 


\section{METHODS}

\section{Subjects}

Eighteen HVs and 13 AA patients completed this study (Table I). HVs had normal baseline lung function $\left(\mathrm{FEV}_{1}\right.$ percent predicted $>80 \%$ and $\mathrm{FEV}_{1} /$ forced vital capacity $[\mathrm{FVC}]$ ratio $>$ 0.75 ), a negative allergy skin test result, ${ }^{22}$ and a negative methacholine challenge result. ${ }^{23}$ AA patients had lung function consistent with no more than mild episodic or mild persistent asthma $\left(\mathrm{FEV}_{1}\right.$ percent predicted $>80 \%$ and $\mathrm{FEV}_{1} / \mathrm{FVC}$ ratio $>0.70$ without use of bronchodilating medications for 12 hours) but a history of episodic wheezing, chest tightness, or shortness of breath after age 6 years consistent with asthma or physician-diagnosed asthma after age 6 years. AA patients also had a positive methacholine test result $\left(\mathrm{PC}_{20}<10 \mathrm{mg} /\right.$ $\mathrm{mL}$ ) and a positive allergy skin test result. See the Methods section in this article's Online Repository at www.jacionline.org for specific details pertaining to skin test procedures. Informed written consent was obtained from all subjects before their participation in the study, which was approved by the Biomedical Institutional Review Board of the University of North Carolina at Chapel Hill. This study was also reviewed and approved by the Data Safety and Monitoring Board of the National Institute of Allergy and Infectious Diseases (NIAID) and the US Food and Drug Administration (IND-BB-9998). The study was listed on clinicaltrials.gov as NCT00839124.

\section{Study design}

All subjects were studied on 5 days: a baseline visit, a prechallenge visit the day before the LPS challenge, an LPS challenge study visit, a 24-hour follow-up visit, and a final visit. During the subject's baseline visit, we measured vital signs, spirometry, and exhaled nitric oxide (eNO) levels, followed by venipuncture and sputum induction. At least 3 days separated the baseline visit and the LPS challenge study visit. After the inhaled LPS challenge, subjects were monitored for spirometry, vital signs, oxygen saturation, and symptom score measurement at the following intervals after challenge: 30 and 60 minutes and then hourly for 3 additional hours. At 6 hours after LPS challenge, we measured eNO levels, followed by venipuncture for blood end points, and sputum induction. See Table E1 in this article's Online Repository at www.jacionline.org for specific procedures related to each study visit.

\section{Inhaled endotoxin challenge}

CCRE (referred to as LPS) was provided by the National Institutes of Health Clinical Center. A dose of 20,000 EU was previously shown to induce sputum neutrophilia and was demonstrated to be safe and well tolerated in previous studies. ${ }^{24}$ Subjects inhaled LPS through a DeVilbiss Ultraneb 99 ultrasonic nebulizer (DeVilbiss Healthcare, Somerset, Pa). Induced sputum was obtained 6 hours after LPS inhalation and processed as previously described. ${ }^{4,25-27}$

\section{Induced sputum assessments}

The procedure for sputum induction has been described in detail previously ${ }^{4}$ and is also included in the Methods section in this article's Online Repository. Subjects had to have previously demonstrated the ability to produce a sputum sample with at least 250,000 cells during a general screening visit. Cell viability (trypan blue exclusion) and total cell counts were assessed in a Neubauer hemocytometer (Hausser Scientific, Horsham, Pa), and differential cell counts were performed on cytocentrifuged cells stained with a modified Wright stain (Hema-Stain-3; Fisher Scientific, Waltham, Mass). Some of the cells were immediately used for flow cytometric assays. 


\section{Flow cytometry}

Expression of selected cell-surface molecules on sputum leukocytes was quantified by using multicolor flow cytometry with a BD LSR-II flow cytometer (BD Immunocytometry Systems, San Jose, Calif), as previously described ${ }^{28}$ These included molecules associated with antigen presentation and specific immunity (CD86/B7.2 and HLA-DR/MHC-II), as well as innate immune function (CD11b/CR3 and CD14/LPS receptor). Upregulation or downregulation of specific surface molecules was quantified as a change in the mean fluorescence intensity of the gated population. Fluorochrome-labeled antibodies were obtained from BD Biosciences (CD11b-phycoerythrin-CY5, CD45-allophycocyanin-Cy7, and HLA-DR-peridinin-chlorophyll-protein complex) and Beckman Coulter (Fullerton, Calif; CD14-allophycocyanin and CD86-phycoerythrin). Appropriate, nonspecific, labeled isotypic control antibodies were also obtained from these sources.

\section{Mediator measurement}

Cytokines from sputum supernatants were measured by using multiplex technology (MesoScaleDiscovery/MSD, Gaithersburg, Md). Each sample was analyzed with the Human TH1/TH2 7-Plex Ultra-Sensitive Kit (IFN- $\gamma$, IL-10, IL-12 p70, IL-13, IL-2, IL-4, and IL-5) and the Human ProInflammatory-4 II Ultra-Sensitive Kit (IL-1 $\beta$, IL-6, IL-8, and TNF- $a$; both from MesoScale-Discovery/MSD). All supernatant samples were diluted 1:4 and had a final dithiothreitol concentration of less than $1 \mathrm{mmol} / \mathrm{L}$, at which no deleterious effects have been observed with the MSD platform. Sputum supernatants were also analyzed for IL-18 by using the Invitrogen (Carlsbad, Calif) Human ELISA IL-18 kit (KHC0181), according to the manufacturer's instructions, with $12.5 \mathrm{pg} / \mathrm{mL}$ as the lowest level of detection.

\section{Exhaled nitric oxide measurement}

Measurements of nitric oxide in the lower airways (exhaled nitric oxide [eNO]) were performed by using an online Sievers Nitric Oxide Analyzer (GE Analytical Instruments, Boulder, Colo). The system is a semiautomatized collection technique using a method recommended by the European Respiratory and the American Thoracic Society (2005). The flow rate was $50 \mathrm{~mL} / \mathrm{s}$.

\section{Conditional power analysis of the primary end point (post-LPS percentage of PMNs)}

This study was designed to test the hypothesis that the AA and HV groups would differ in the degree of neutrophilic inflammation present in sputum (expressed as the percentage of neutrophils in sputum [\% PMN] as the primary end point) after LPS challenge. Our initial sample size estimate was based on measures of variability of the \%PMN from prior studies of AA and HV cohorts using different sources and doses of LPS, which indicated that 18 in each group would provide adequate statistical power for this study. A conditional power analysis was planned after $10 \mathrm{HVs}$ and $10 \mathrm{AA}$ patients had been recruited to ensure that our initial estimate was appropriate. However, HV recruitment was already completed $(n=18)$ when sputum data from AA volunteers 9 to 13 (providing $n \geq 10 \mathrm{AA}$ ) became contemporaneously available, resulting in an interim analysis based on data from $18 \mathrm{HVs}$ and 13 HVs. The conditional power analysis is described in the Results section in this article's Online Repository at www.jacionline.org, and after review by the NIAID's Data Safety Monitoring Board, recruitment was suspended with 18 HVs and 13 AA volunteers.

\section{Statistical analysis of other sputum end points}

For all responses, including \%PMNs, the cellular response to LPS expressed as the change from baseline in total leukocyte numbers (cells per milligram), neutrophil response (PMNs per milligram) in sputum, and the remaining exploratory measures, such as circulating cell counts, sputum cytokine concentrations, and cell-surface marker expression of sputum cells, 
we used the following strategy to assess the LPS challenge effects. The effects within a group (either HVs or AA patients) were evaluated through paired testing of the difference between the baseline values (pre-LPS challenge values) and post-LPS challenge values. The effects of LPS challenge between the AA and HV groups were evaluated by using a 2sample test comparing the postchallenge-prechallenge difference in AA patients with the postchallenge-prechallenge differences in HVs. Proper transformations to those responses were conducted to normalize the data. The reported results are based on nonparametric paired $t$ tests (the Wilcoxon signed-rank rest) for the changes within each group and nonparametric $t$ tests (the Mann-Whitney test) for between-group comparisons at the nominal level of .05 .

\section{RESULTS}

\section{Subjects' demographics}

Twenty-one HVs and 17 AA patients were enrolled in this study. Of these, 18 HVs and 13 patients with mild intermittent AA completed the study. HVs were withdrawn for abnormal baseline complete blood cell counts with differential, recent viral illness, or smoking. AA patients were withdrawn for abnormal baseline spirometric results $\left(\mathrm{FEV}_{1} / \mathrm{FVC}\right.$ ratio $\left.<0.70\right)$, hypertension, or baseline abnormal complete blood cell counts with differential. Demographics for those completing the study are shown in Table I. All subjects who completed the study tolerated the inhaled LPS challenge without significant changes in vital signs, symptom scores, or decrements in spirometric results (data not shown).

\section{Sputum neutrophilia (\%PMNs)}

The primary end point for this study was the \%PMN present after LPS challenge, with our original expectation that AA patients would have increased sputum neutrophilia after LPS challenge compared with that seen in HVs. We found that \%PMNs significantly increased in both the HV and AA groups after LPS challenge; however, the post-LPS challenge \%PMNs were significantly lower in the AA patients compared with the HVs (Fig 1). Baseline $\%$ PMNs of AA patients were also lower than those of HVs $(P=.06)$. However, there was no significant difference between groups $(P=.9522)$ in \%PMNs that increased with LPS challenge (post-LPS minus pre-LPS \%PMNs).

\section{Other sputum and circulating cellularity end points}

Although \%PMNs increased after LPS challenge in both groups, the total number of cells in sputum (expressed as cells per milligram of sputum; Fig 2, $A$ ) and neutrophils in sputum (expressed as PMNs per milligram of sputum; Fig 2, B) were increased only in the HV group after LPS challenge. To further characterize the degree of the cellular response, we compared the difference between the post-LPS cell count and the baseline cell count (postLPS minus pre-LPS values) between the cohorts, finding that HVs had significantly greater increases in both total sputum cell $(P=.0009)$ and neutrophil $(P=.003)$ numbers than AA patients after inhaled LPS challenge. eNO values and numbers of eosinophils per milligram at baseline were significantly increased in the AA patients compared with the HVs (Table I) but were not changed with LPS challenge.

We next examined the response of circulating cells to determine whether there was a systemic difference between the 2 cohorts consistent with the difference in induced sputum responses with LPS challenge. Both cohorts significantly increased their total white blood cell (WBC) counts (Fig 2, C) and absolute neutrophil counts (Fig 2,D) after inhaled LPS challenge. However, the baseline WBC and absolute neutrophil counts in this cohort of AA patients was lower compared with the baseline WBC $(P=.01)$ and absolute neutrophil $(P=$. 004) counts in HVs. We also found decreased absolute neutrophil numbers in the blood after 
LPS challenge in AA patients compared with HVs $(P=.04)$. This result suggests that this cohort of AA patients responded systemically to LPS, although this response is not as robust as that seen in HVs.

We randomly selected 5 samples each from the HV and AA cohorts for LPS assessments of induced sputum (pre-LPS vs post-LPS) by using a limulus assay performed at our tissue culture facility. We found no significant differences between the HVs and AA patients in either baseline levels of LPS or post-CCRE challenge LPS levels (data not shown).

\section{Induced sputum cytokines}

We next explored why the AA cohort had decreased induced sputum cell and neutrophil counts compared with those seen in HVs by examining cytokine concentrations in the sputum super-natant samples that represent LPS signaling through TLR4. We found that both HVs and AA patients had significant increases in both TNF- $a$ and IL-6 concentrations in induced sputum after LPS challenge (Fig 3, $A$ and $B$ ). However, only HVs had significant increases in levels of the inflammasome-dependent cytokines IL-1 $\beta$ and IL-18 with LPS challenge (Fig 3, $C$ and $D$ ). There were no differences with LPS challenge in either cohort in levels of IL-8, IL-4, IL-5, or IL-13 (data not shown). These data suggest that LPS signaling through TLR4 is preserved in both groups but that inflammasome-dependent processing of IL-1 $\beta$ and IL-18 might be impaired in AA patients after inhaled LPS challenge.

\section{Macrophage surface marker expression}

LPS is known to interact with TLR4 and CD14 to initiate proinflammatory cytokine signaling. We have previously shown increased surface marker expression of CD14 in HVs and AA patients after inhaled LPS challenge. ${ }^{24,27}$ We examined surface marker expression on both induced sputum monocytes (thought to be newly recruited cells to the airway) and macrophages in $13 \mathrm{HVs}$ and $7 \mathrm{AA}$ patients. We found no significant change in monocyte TLR4 surface expression with LPS challenge (Fig 4, $A$ ) but did find significant increases in monocyte CD14 abundance after LPS challenge in both groups (Fig 4, B). In macrophages we found increases in TLR4, TLR2, and CD11b levels (Fig 4, C-F) in HVs but not in AA patients. We found no significant changes in macrophage CD14 expression with LPS challenge in either cohort (Fig 4,D). We also noted increased (although not significantly) surface expression of baseline TLR4 in monocytes and macrophages of AA patients and significantly increased expression of TLR2 on sputum macrophages of AA patients (Fig 4, E).

\section{DISCUSSION}

In this open-label study $18 \mathrm{HVs}$ and $13 \mathrm{AA}$ patients underwent an inhaled LPS challenge with 20,000 EU of CCRE. We originally hypothesized that AA patients would have exaggerated inflammatory responses to LPS compared with HVs. Previous data suggested that (1) AA patients have increased levels of CD14 after inhaled allergen challenge ${ }^{29}$; (2) LPS induces airway neutrophilia, correlating with increased levels of CD14 expression on airway macrophages ${ }^{8}$; and (3) CD14 expression correlates with eosinophil numbers in the airways ${ }^{8}$ and pretreatment of allergic inflammation with fluticasone decreases surface monocyte CD14 levels and PMN responses to 20,000 EU CCRE. ${ }^{8}$ After inhaled LPS challenge, both cohorts showed increases in \%PMNs recruited to the airway. The final $\%$ PMNs present after LPS challenge were significantly less in AA patients than in HVs, but AA patients had reduced \%PMNs at baseline compared with those seen in HVs, with no significant difference in the \%PMNs recruited to the airway when comparing HVs and AA patients. There was augmentation of the proinflammatory cytokines IL- 6 and TNF- $\alpha$ and increases in sputum monocyte CD14 expression in both groups. 
However, there were clear differences between HVs and AA patients when examining the absolute numbers of cells (cells per milligram of sputum) recruited to the airway with LPS challenge. AA patients had increased airway eosinophilic inflammation at baseline, which was not significantly modified by LPS challenge. Compared with HVs, AA patients had reduced numbers of total leukocytes and neutrophils recovered from induced sputum with inhaled LPS challenge. AA patients also had decreased numbers of WBCs and neutrophils in the peripheral blood at baseline, as well as decreased post-LPS WBC and PMN counts. In AA patients decreases in circulating leukocyte numbers at baseline might account for decreased airway PMN counts after LPS challenge. TLR4/CD14-mediated signaling appears to be similar in the AA and HV groups because they mounted similar sputum TNF-a and IL-6 responses with LPS. However, we could not detect significant increases in the levels of IL-1 $\beta$ or IL-18 in sputum from AA patients in contrast to increases seen in HVs. Recent data from our group demonstrated decreased inflammasome gene expression in sputum inflammatory cells from patients with atopic asthma compared with HVs. ${ }^{30}$ Further examination of induced sputum macrophages with larger sample sizes will have to be performed to determine whether AA patients produce less pro-IL-1 $\beta$ and pro-IL-18 than $\mathrm{HV}$, whether activation of the $\mathrm{P} 2 \mathrm{X} 7$ receptor (that then activates the inflammasome) is altered in AA patients after LPS challenge, and/or whether cleavage of these proforms into the mature soluble forms is impaired in AA patients compared with HVs. Previous work by our group did show a 16 -fold increase in pro-IL-1 $\beta$ mRNA in an allergic cohort consisting of both asthmatic and nonasthmatic subjects after inhaled LPS challenge with 10,000 EU CCRE, suggesting that inflammasome function might be impaired in atopic patients. ${ }^{11}$

IL- $1 \beta$ is produced in response to various inflammatory stimuli and likely plays a central role in mediating neutrophilic airway responses in human subjects. IL- $1 \beta$ is involved in the recruitment of airway neutrophils by LPS in murine models 31,32 and intratracheal administration of IL-1 receptor antagonist inhibits LPS-induced airway neutrophilia in mice. ${ }^{32}$ Our data indicate that decreased numbers of airway neutrophils recovered from induced sputum in AA patients after LPS challenge in part might be due to the lack of IL-1 $\beta$ production by AA patients, although we cannot exclude other factors, such as expression of intercellular adhesion molecules that regulate chemotaxis to the airways.

This IL-1 $\beta$ response in AA patients was unexpected because recent gene-association studies have indicated a significant role for IL-1 $\beta$ in asthma. ${ }^{33}$ In human subjects IL-1 $\beta$ has been reported to be increased in bronchoalveolar lavage fluid and sputum of asthmatic patients compared with $\mathrm{HVs},{ }^{34}$ with airway macrophages from asthmatic patients displaying increased expression of IL- $1 \beta .{ }^{35} \mathrm{IL}-1 \beta$ levels are also increased in broncho-alveolar lavage fluid from persons with symptomatic asthma versus those with asymptomatic asthma, ${ }^{36}$ and there is increased expression of this cytokine in the airways epithelium of asthmatic patients. ${ }^{37}$ We have shown that after ozone exposure, AA patients have increased levels of sputum IL- $1 \beta$ in their airways, whereas HVs do not. ${ }^{22}$ However, these differences might be attributable to the site of injury that triggers the inflammatory response. Most asthma exacerbations are caused by viral infections that damage the airway epithelium. Ozone exposure and other agents of environmental pollution also induce epithelial cell oxidative stress. In contrast, inhaled LPS directly activates airway macrophages through interactions with TLR4 and CD14. We hypothesize that the initial cellular target of injury might influence the asthmatic response, with increased inflammation after epithelial cell injury and reduced inflammation if injury to the epithelium is bypassed.

Leukocytes from atopic asthmatic patients might be less responsive to TLR stimuli compared with those from nonatopic subjects. Peripheral blood monocytes from atopic children exhibited reduced upregulation of TLR2 after ex vivo peptidoglycan stimulation compared with responses from monocytes of nonatopic children. ${ }^{38}$ Likewise, signaling 
through TLR4 was recently noted to be impaired in $\mathrm{CD} 4^{+}$monocytes derived from atopic children compared with nonatopic children. ${ }^{39}$ In adult populations asthmatic patients had reduced production of TNF- $\alpha$, IL-10, and IL- $1 \beta$ after $e x$ vivo stimulation by LPS and flagellin compared with nonasthmatic patients. ${ }^{40}$ These studies are limited in that PBMCs might have differential expression of TLRs than those found in the airway, with Juarez et $\mathrm{al}^{41}$ showing differential responses to various TLR ligands in alveolar macrophages compared with those seen in peripheral monocytes. After ex vivo LPS exposure, alveolar macrophages from asthmatic patients (approximately one half were atopic) did not show significantly increased IL- $1 \beta$ production, whereas those from HVs did. ${ }^{35}$ It is still unclear whether airway macrophages from asthmatic patients and other resident leukocytes, such as eosinophils, are less responsive to TLR activation, thus conferring increased risk to airway infections. Alternatively, the specific milieu of inflammatory cells that reflect underlying immune dysregulation, such as the increased eosinophil numbers noted in asthmatic patients at baseline, might alter responses to infection. In mice infected with Haemophilus influenzae, it was recently found that those with allergic airways disease had chronic lung infection compared with nonallergic mice and that airway influx, development of phagocytosing neutrophils and macrophages, or both were reduced. ${ }^{42}$ It remains unclear whether atopic status, asthma status, or both contribute to the increased susceptibility to airway infections noted in asthmatic patients and which cell types are responsible for this susceptibility.

In conclusion, although we show that the percentage of neutrophils increases in both HVs and AA patients after LPS challenge, the absolute numbers of inflammatory cells recruited to the airway (cells per milligram of sputum) and circulation (cells per deciliter) are significantly less in AA patients compared with those in HVs. Although we could detect similar and significant induction of TLR4-MyD88-regulated proinflammatory cytokines, such as IL- 6 and TNF-a, in the sputum supernatants of both groups, AA patients had muted IL-1 $\beta$ and IL-18 responses to LPS. These data, coupled with those of our prior report, are consistent with the hypothesis that atopic asthmatic patients have modified innate immune responses characterized by decreased phagocytic inflammasome function. We hypothesize that the development of $\mathrm{T}_{\mathrm{H}} 2$ inflammation results in downregulation of inflammasome components of phagocytes in atopic subjects. Although the innate immune response is important in mediating exacerbation of asthma, downregulation of elements of innate immunity by the $\mathrm{T}_{\mathrm{H}} 2$ immune response likely contributes to the increased occurrence of invasive microbial infections observed in this group, as well as changes in microbiome populations reported in atopic populations that modify asthma severity.

\section{METHODS}

\section{Skin prick testing}

Atopy was demonstrated by a positive immediate skin test response to one of the following allergen mixes: 2 species of house dust mite (Dermatophagoides farinae and Dermatophagoides pteronyssinus), cockroach, tree mix, grass mix, weed mix, mold mix 1 , mold mix 2, rat, mouse, guinea pig, rabbit, cat, or dog. A positive skin test response was defined as a wheal $3 \mathrm{~mm}$ larger than that elicited by the negative saline control.

\section{Sputum induction and processing}

For sputum induction, three 7-minute inhalation periods of nebulized hypertonic saline (3\%, 4\%, and 5\%; DeVilbiss UltraNeb 99 ultrasonic nebulizer) were followed by expectoration of sputum into a sterile specimen cup. A cell-enriched "select" sample was obtained by selecting visible clumps of cells and cell-rich mucus plugs from the raw sample to separate them from noncellular portions of the sample. This was then treated with a dilute $(0.1 \%)$ 
solution of dithiothreitol (Sputolysin; Calbiochem, San Diego, Calif) in Dulbecco PBS to depolymerize mucins. After centrifugation ( $500 g$ for 10 minutes), aliquots of sputum supernatants were immediately frozen and stored at $-80^{\circ} \mathrm{C}$, and the cell pellet was suspended in a small volume of Hanks balanced salt solution. Cell viability (trypan blue exclusion) and total cell counts were assessed by using a Neubauer hemocytometer, and differential cell counts were performed on cytocentrifuged cells stained with a modified Wright stain (Hema-Stain-3, Fisher Scientific). Some of the cells were immediately used for flow cytometric assays.

\section{RESULTS}

The primary end point for this study was the \%PMN after LPS challenge, with our original expectation that AA patients would have increased sputum neutrophilia after LPS challenge compared with HVs. To determine whether we could cease recruitment of AA patients after enrolling 13 AAvolunteers and $18 \mathrm{HVs}$, we undertook a conditional power analysis focused on the differences between post-LPS \%PMNs of the 13 AA patients and 18 HVs. Using procedures outlined for 2-arm trials with arcsin transformation ${ }^{\mathrm{E} 1}$ of post-LPS \%PMNs to ensure normal distribution of these data, we calculated a conditional power of 0.9827 based on the sample pooled SD $\sigma$ of 0.2403 and sample mean difference at 0.225 , suggesting that continued recruitment of AA patients (assuming similar variation in sputum PMN data) would not yield a different result. We also undertook a conditional power analysis based on the postchallenge-prechallenge difference in \%PMNs between the HVand AA groups. We calculated a conditional power close to $0(2.31 \mathrm{E}-6)$ based on the sample pooled SD $\sigma$ of 28.33 and sample mean difference at 0.5596 , suggesting that the study be stopped for futility if the postchallenge-prechallenge difference in \%PMNs was the main end point. These findings were presented to the NIAID's Data Safety Monitoring Board, and although this analysis had not been conducted as originally planned, the Data Safety Monitoring Board granted permission to halt recruitment of additional AA volunteers and proceed with analysis of all other exploratory end points.

\section{Acknowledgments}

We thank Martha Almond, Carole Robinette, and Aline Kala for their assistance in study-related procedures.

M.L.H. is supported by National Institutes of Health grant KL2RR025746. N.E.A., W.J.B., J.P.Y.T., and D.B.P. are supported by National Institute of Allergy and Infectious Diseases grant U19AI077437. This work was also supported by CTSA UL1RR025747.

\section{Abbreviations used}

$\begin{array}{ll}\text { AA } & \text { Allergic asthmatic } \\ \text { CCRE } & \text { Clinical Center Reference Endotoxin } \\ \text { eNO } & \text { Exhaled nitric oxide } \\ \text { EU } & \text { Endotoxin units } \\ \text { FVC } & \text { Forced vital capacity } \\ \text { HV } & \text { Healthy volunteer } \\ \text { NIAID } & \text { National Institute of Allergy and Infectious Diseases } \\ \text { \%PMN } & \text { Percentage of neutrophils in sputum } \\ \text { TLR } & \text { Toll-like receptor }\end{array}$


WBC White blood cell

\section{REFERENCES}

1. Gern JE, Busse WW. Relationship of viral infections to wheezing illnesses and asthma. Nat Rev Immunol. 2002; 2:132-8. [PubMed: 11910895]

2. Peden DB. The epidemiology and genetics of asthma risk associated with air pollution. J Allergy Clin Immunol. 2005; 115:213-20. [PubMed: 15696070]

3. Zeldin DC, Eggleston P, Chapman M, Piedimonte G, Renz H, Peden D. How exposures to biologics influence the induction and incidence of asthma. Environ Health Perspect. 2006; 114:620-6. [PubMed: 16581556]

4. Alexis NE, Lay JC, Zeman K, Bennett WE, Peden DB, Soukup JM, et al. Biological material on inhaled coarse fraction particulate matter activates airway phagocytes in vivo in healthy volunteers. J Allergy Clin Immunol. 2006; 117:1396-403. [PubMed: 16751003]

5. Becker S, Fenton MJ, Soukup JM. Involvement of microbial components and toll-like receptors 2 and 4 in cytokine responses to air pollution particles. Am J Respir Cell Mol Biol. 2002; 27:611-8. [PubMed: 12397021]

6. Mueller-Anneling L, Avol E, Peters JM, Thorne PS. Ambient endotoxin concentrations in PM10 from Southern California. Environ Health Perspect. 2004; 112:583-8. [PubMed: 15064165]

7. Thorne PS, Kulhankova K, Yin M, Cohn R, Arbes SJ Jr, Zeldin DC. Endotoxin exposure is a risk factor for asthma: the national survey of endotoxin in United States housing. Am J Respir Crit Care Med. 2005; 172:1371-7. [PubMed: 16141442]

8. Alexis N, Eldridge M, Reed W, Bromberg P, Peden DB. CD14-dependent airway neutrophil response to inhaled LPS: role of atopy. J Allergy Clin Immunol. 2001; 107:31-5. [PubMed: 11149987]

9. Michel O, Duchateau J, Sergysels R. Effect of inhaled endotoxin on bronchial reactivity in asthmatic and normal subjects. J Appl Physiol. 1989; 66:1059-64. [PubMed: 2708231]

10. Michel O, Ginanni R, Le Bon B, Content J, Duchateau J, Sergysels R. Inflammatory response to acute inhalation of endotoxin in asthmatic patients. Am Rev Respir Dis. 1992; 146:352-7. [PubMed: 1489124]

11. Alexis NE, Brickey WJ, Lay JC, Wang Y, Roubey RA, Ting JP, et al. Development of an inhaled endotoxin challenge protocol for characterizing evoked cell surface phenotype and genomic responses of airway cells in allergic individuals. Ann Allergy Asthma Immunol. 2008; 100:20615. [PubMed: 18426139]

12. Michel O, Ginanni R, Sergysels R. Relation between the bronchial obstructive response to inhaled lipopolysaccharide and bronchial responsiveness to histamine. Thorax. 1992; 47:288-91. [PubMed: 1585294]

13. Martin RJ, Kraft M, Chu HW, Berns EA, Cassell GH. A link between chronic asthma and chronic infection. J Allergy Clin Immunol. 2001; 107:595-601. [PubMed: 11295645]

14. Webley WC, Salva PS, Andrzejewski C, Cirino F, West CA, Tilahun Y, et al. The bronchial lavage of pediatric patients with asthma contains infectious Chlamydia. Am J Respir Crit Care Med. 2005; 171:1083-8. [PubMed: 15735056]

15. Sutherland ER, Martin RJ. Asthma and atypical bacterial infection. Chest. 2007; 132:1962-6. [PubMed: 18079229]

16. Juhn YJ, Kita H, Yawn BP, Boyce TG, Yoo KH, McGree ME, et al. Increased risk of serious pneumococcal disease in patients with asthma. J Allergy Clin Immunol. 2008; 122:719-23. [PubMed: 18790525]

17. Jung JA, Kita H, Yawn BP, Boyce TG, Yoo KH, McGree ME, et al. Increased risk of serious pneumococcal disease in patients with atopic conditions other than asthma. J Allergy Clin Immunol. 2010; 125:217-21. [PubMed: 20109748]

18. Klemets P, Lyytikainen O, Ruutu P, Ollgren J, Kaijalainen T, Leinonen M, et al. Risk of invasive pneumococcal infections among working age adults with asthma. Thorax. 2010; 65:698-702. [PubMed: 20685743] 
19. Talbot TR, Hartert TV, Mitchel E, Halasa NB, Arbogast PG, Poehling KA, et al. Asthma as a risk factor for invasive pneumococcal disease. N Engl J Med. 2005; 352:2082-90. [PubMed: 15901861]

20. Huang YJ, Nelson CE, Brodie EL, Desantis TZ, Baek MS, Liu J, et al. Airway microbiota and bronchial hyperresponsiveness in patients with suboptimally controlled asthma. J Allergy Clin Immunol. 2011; 127:372-81. e1-3. [PubMed: 21194740]

21. Hilty M, Burke C, Pedro H, Cardenas P, Bush A, Bossley C, et al. Disordered microbial communities in asthmatic airways. PLoS One. 2010; 5:e8578. [PubMed: 20052417]

22. Hernandez ML, Lay JC, Harris B, Esther CR Jr, Brickey WJ, Bromberg PA, et al. Atopic asthmatic subjects but not atopic subjects without asthma have enhanced inflammatory response to ozone. $\mathrm{J}$ Allergy Clin Immunol. 2010; 126:537-44. e1. [PubMed: 20816188]

23. Crapo RO, Casaburi R, Coates AL, Enright PL, Hankinson JL, Irvin CG, et al. Guidelines for methacholine and exercise challenge testing-1999. This official statement of the American Thoracic Society was adopted by the ATS Board of Directors, July 1999. Am J Respir Crit Care Med. 2000; 161:309-29. [PubMed: 10619836]

24. Hernandez ML, Harris B, Lay JC, Bromberg PA, Diaz-Sanchez D, Devlin RB, et al. Comparative airway inflammatory response of normal volunteers to ozone and lipopolysaccharide challenge. Inhal Toxicol. 2010; 22:648-56. [PubMed: 20540623]

25. Alexis NE, Zhou H, Lay JC, Harris B, Hernandez ML, Lu TS, et al. The glutathione-S-transferase Mu 1 null genotype modulates ozone-induced airway inflammation in human subjects. J Allergy Clin Immunol. 2009; 124:1222-8. e5. [PubMed: 19796798]

26. Lay JC, Alexis NE, Kleeberger SR, Roubey RA, Harris BD, Bromberg PA, et al. Ozone enhances markers of innate immunity and antigen presentation on airway monocytes in healthy individuals. J Allergy Clin Immunol. 2007; 120:719-22. [PubMed: 17586033]

27. Alexis NE, Eldridge MW, Peden DB. Effect of inhaled endotoxin on airway and circulating inflammatory cell phagocytosis and CD11b expression in atopic asthmatic subjects. J Allergy Clin Immunol. 2003; 112:353-61. [PubMed: 12897742]

28. Lay JC, Peden DB, Alexis NE. Flow cytometry of sputum: assessing inflammation and immune response elements in the bronchial airways. Inhal Toxicol. 2011; 23:392-406. [PubMed: 21639708]

29. Lensmar C, Katchar K, Eklund A, Grunewald J, Wahlstrom J. Phenotypic analysis of alveolar macrophages and lymphocytes following allergen inhalation by atopic subjects with mild asthma. Respir Med. 2006; 100:918-25. [PubMed: 16202577]

30. Brickey WJ, Alexis NE, Hernandez ML, Reed W, Ting JP, Peden DB. Sputum inflammatory cells from patients with allergic rhinitis and asthma have decreased inflammasome gene expression. $\mathbf{J}$ Allergy Clin Immunol. 2011; 128:900-3. [PubMed: 21868073]

31. Ulich TR, Watson LR, Yin SM, Guo KZ, Wang P, Thang H, et al. The intratracheal administration of endotoxin and cytokines. I. Characterization of LPS-induced IL-1 and TNF mRNA expression and the LPS-, IL-1-, and TNF-induced inflammatory infiltrate. Am J Pathol. 1991; 138:1485-96. [PubMed: 2053596]

32. Ulich TR, Yin SM, Guo KZ, del Castillo J, Eisenberg SP, Thompson RC. The intratracheal administration of endotoxin and cytokines. III. The interleukin-1 (IL-1) receptor antagonist inhibits endotoxin- and IL-1-induced acute inflammation. Am J Pathol. 1991; 138:521-4. [PubMed: 1825745]

33. Ober C, Hoffjan S. Asthma genetics 2006: the long and winding road to gene discovery. Genes Immun. 2006; 7:95-100. [PubMed: 16395390]

34. Konno S, Gonokami Y, Kurokawa M, Kawazu K, Asano K, Okamoto K, et al. Cytokine concentrations in sputum of asthmatic patients. Int Arch Allergy Immunol. 1996; 109:73-8. [PubMed: 8527954]

35. Pujol JL, Cosso B, Daures JP, Clot J, Michel FB, Godard P. Interleukin-1 release by alveolar macrophages in asthmatic patients and healthy subjects. Int Arch Allergy Appl Immunol. 1990; 91:207-10. [PubMed: 2341202]

36. Broide DH, Lotz M, Cuomo AJ, Coburn DA, Federman EC, Wasserman SI. Cytokines in symptomatic asthma airways. J Allergy Clin Immunol. 1992; 89:958-67. [PubMed: 1374772] 
37. Sousa AR, Lane SJ, Nakhosteen JA, Lee TH, Poston RN. Expression of interleukin-1 beta (IL-1beta) and interleukin-1 receptor antagonist (IL-1ra) on asthmatic bronchial epithelium. Am J Respir Crit Care Med. 1996; 154:1061-6. [PubMed: 8887608]

38. Amoudruz P, Holmlund U, Saghafian-Hedengren S, Nilsson C, Sverremark-Ekstrom E. Impaired Toll-like receptor 2 signalling in monocytes from 5-year-old allergic children. Clin Exp Immunol. 2009; 155:387-94. [PubMed: 19220829]

39. Prefontaine D, Banville-Langelier AA, Fiset PO, Guay J, An J, Mazer M, et al. Children with atopic histories exhibit impaired lipopolysaccharide-induced Toll-like receptor-4 signalling in peripheral monocytes. Clin Exp Allergy. 2010; 40:1648-57. [PubMed: 20636402]

40. Lun SW, Wong CK, Ko FW, Hui DS, Lam CW. Expression and functional analysis of toll-like receptors of peripheral blood cells in asthmatic patients: implication for immunopathological mechanism in asthma. J Clin Immunol. 2009; 29:330-42. [PubMed: 19067129]

41. Juarez E, Nunez C, Sada E, Ellner JJ, Schwander SK, Torres M. Differential expression of Tolllike receptors on human alveolar macrophages and autologous peripheral monocytes. Respir Res. 2010; 11:2. [PubMed: 20051129]

42. Essilfie AT, Simpson JL, Dunkley ML, Morgan LC, Oliver BG, Gibson PG, et al. Combined Haemophilus influenzae respiratory infection and allergic airways disease drives chronic infection and features of neutrophilic asthma. Thorax. 2012 [Epub ahead of print].

E1. Proschan, MA.; Turk Wittes, J. Statistical monitoring of clinical trials: a unified approach. Springer; Berlin: 2006. 


\section{Clinical implications}

Our findings suggest that AA patients have reduced innate immune airway inflammatory responses after LPS inhalation compared with HVs. This might increase susceptibility to infection in AA patients. 


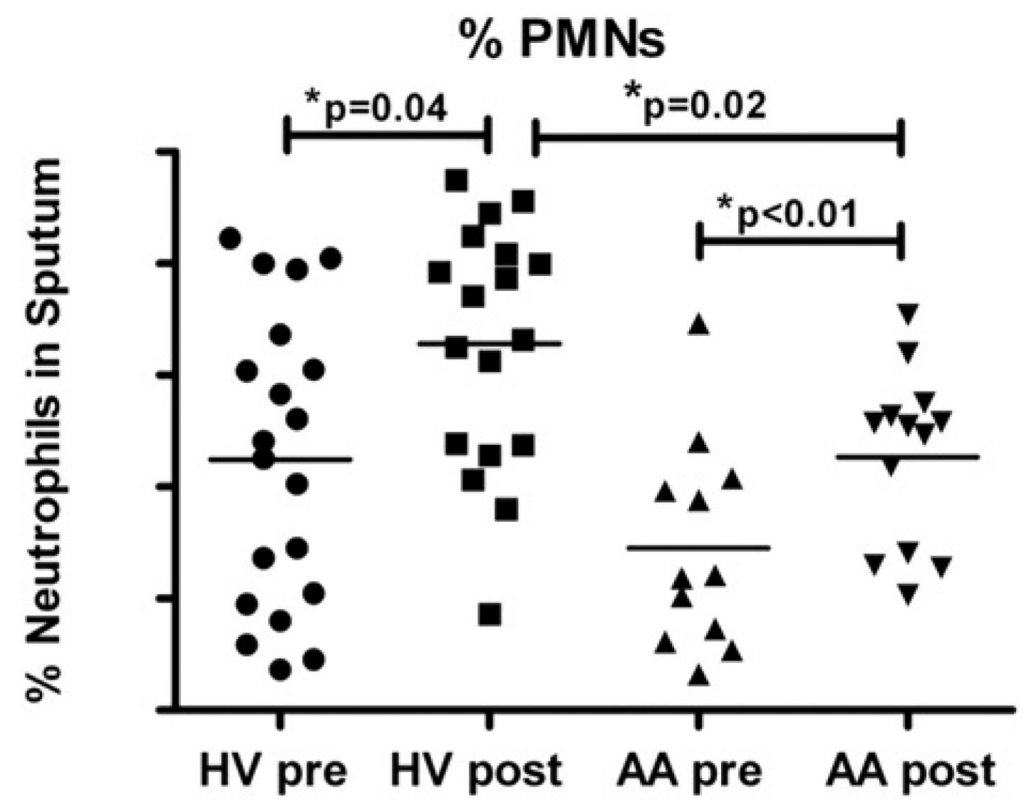

FIG 1.

\%PMNs in sputum before and after inhaled LPS challenge. \%PMNs significantly increased in both cohorts, with no significant difference in post-LPS-pre-LPS \%PMNs between groups. Lines represent means ( $\mathrm{n}=18 \mathrm{HVs}$ and $13 \mathrm{AA}$ patients). 

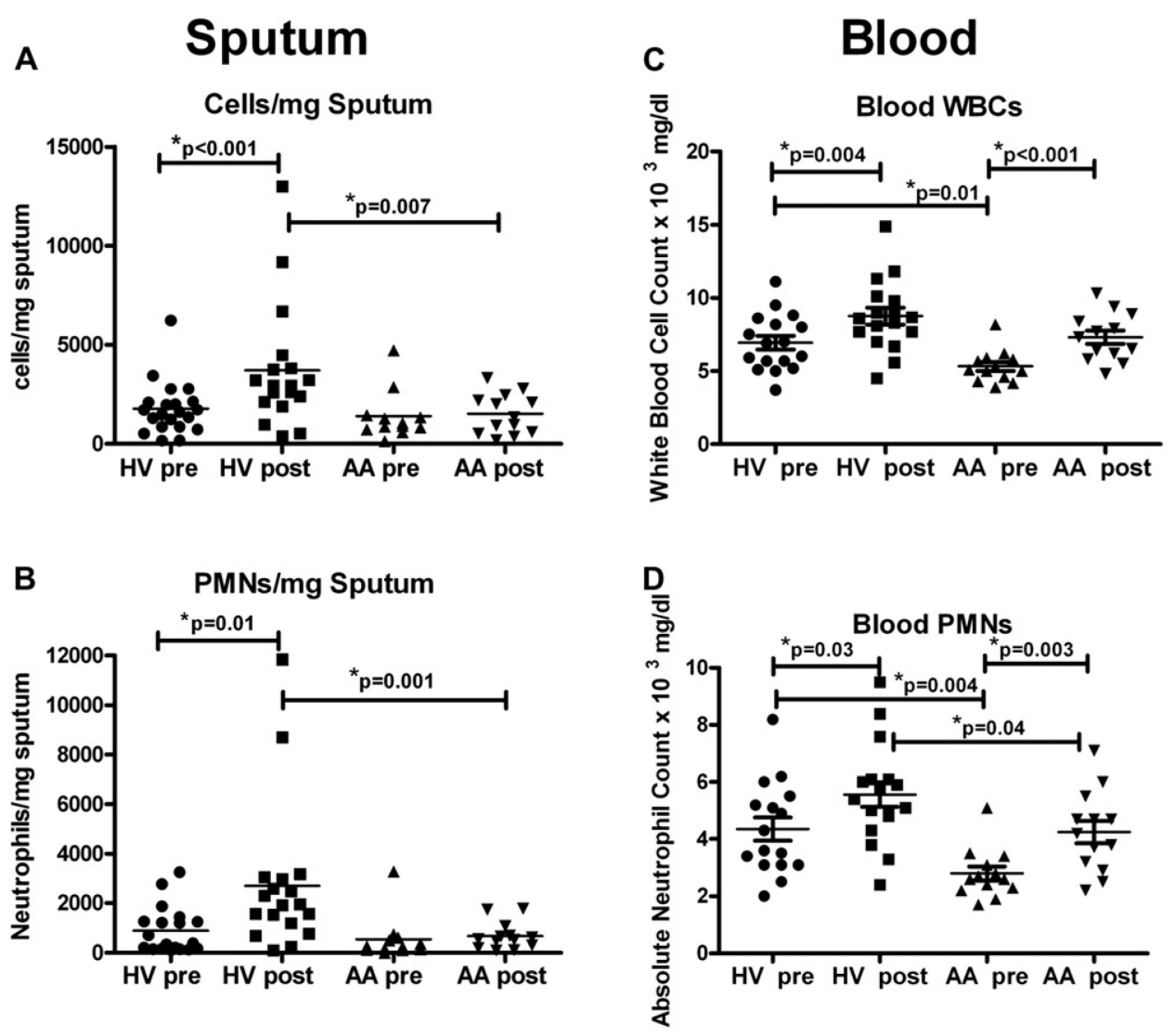

FIG 2.

Leukocyte numbers in induced sputum and peripheral blood. Sputum, Absolute numbers of total leukocytes (cells per milligram, A) and neutrophils (PMNs per milligram, B) in induced sputum before (pre-LPS) and after (post-LPS) challenge. Blood, Total numbers of WBCs $(\mathbf{C})$ and PMNs (D) in the blood of HVs and AA patients with LPS challenge. Lines represent means ( $\mathrm{n}=18 \mathrm{HVs}$ and 13 AA patients). 

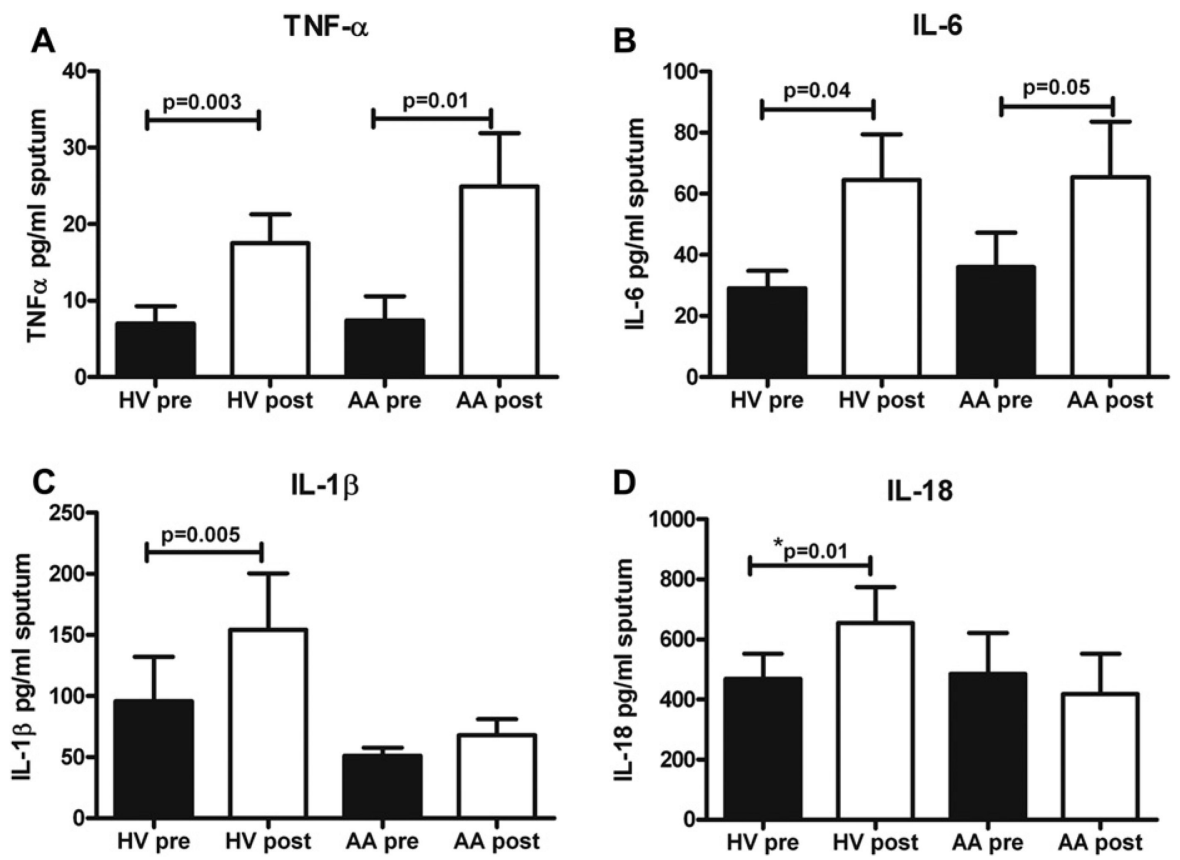

FIG 3.

Proinflammatory cytokines recovered from induced sputum. Mean concentrations of TNF-a (A), IL-6 (B), IL-1 $\beta$ (C), and IL-18 (D) measured in induced sputum supernatants before LPS and after LPS challenge are shown. Means and SEMs are shown $(\mathrm{n}=18 \mathrm{HVs}$ and 12 AA patients). 

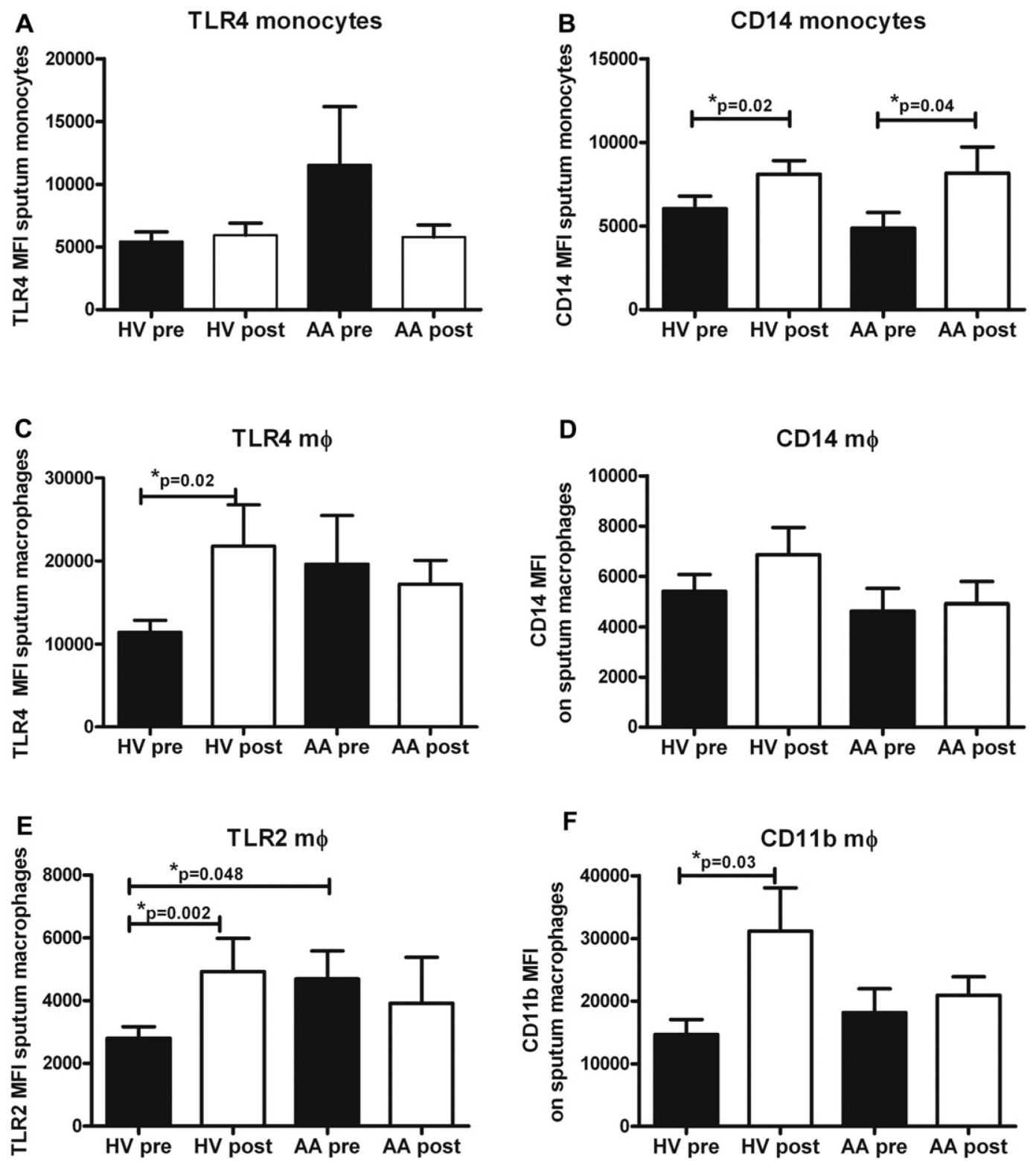

FIG 4.

Monocyte and macrophage surface marker expression from induced sputum. Monocytes, Mean fluorescence intensity (MFI) of TLR4 (A) and CD14 (B) before and after LPS challenge in HVs and AA patients. Macrophages $(m \Phi)$, MFI of TLR4 (C), CD14 (D), TLR2 $(\mathbf{E})$, and CD11b $(\mathbf{F})$ before and after LPS challenge. Means with SEMs are shown $(\mathrm{n}=13$ HVs and 7 AA patients). 


\section{TABLE I}

Characteristics of participants

\begin{tabular}{|c|c|c|}
\hline Clinical characteristic & HVs & AA patients \\
\hline No. & 18 & 13 \\
\hline Age (y) & $24.5 \pm 6.4$ & $26.6 \pm 8.1$ \\
\hline Sex & $12 \mathrm{~F} / 6 \mathrm{M}$ & $8 \mathrm{~F} / 5 \mathrm{M}$ \\
\hline \multirow[t]{3}{*}{ Race } & 13 White & 8 White \\
\hline & 5 African American & 4 African American \\
\hline & & 1 Asian \\
\hline BMI $\left(\mathrm{kg} / \mathrm{m}^{2}\right)$ & $27.2 \pm 6.4$ & $24.3 \pm 2.7$ \\
\hline \multicolumn{3}{|l|}{ Baseline spirometry (\% predicted) } \\
\hline $\mathrm{FVC}$ & $106.7 \pm 11.7$ & $102.1 \pm 12.1$ \\
\hline $\mathrm{FEV}_{1}$ & $102.4 \pm 12.6$ & $94.5 \pm 11.7$ \\
\hline \multicolumn{3}{|l|}{ Medications (no. of subjects) } \\
\hline Antihistamines & 0 & $3^{*}$ \\
\hline Leukotriene receptor antagonists & 0 & 0 \\
\hline Nasal steroids & 0 & 0 \\
\hline Inhaled steroids & 0 & 0 \\
\hline Short-acting bronchodilator as necessary & 0 & 13 \\
\hline \multicolumn{3}{|c|}{ Skin prick test response positivity (no. of subjects) } \\
\hline Dust mites & 0 & 11 \\
\hline Trees & 0 & 8 \\
\hline Grasses & 0 & 9 \\
\hline Weeds & 0 & 8 \\
\hline Cat/dog & 0 & 8 \\
\hline Cockroach & 0 & 4 \\
\hline \multicolumn{3}{|l|}{ eNO (nL/min) } \\
\hline Before LPS & $11.9 \pm 9.1$ & $66.9 \pm 66.2^{\dagger}$ \\
\hline Six hours after LPS & $14.7 \pm 11.7$ & $64.3 \pm 64.2$ \\
\hline \multicolumn{3}{|l|}{ Sputum eosinophils/mg } \\
\hline Before LPS & $4.4 \pm 7.7$ & $34.4 \pm 32.9^{\dagger}$ \\
\hline Six hours after LPS & $11.7 \pm 12.0$ & $47.2 \pm 73.9$ \\
\hline
\end{tabular}

Values are presented as means \pm SDs where shown.

$B M I$, Body mass index; $F$, female; $M$, male.

* Subjects had to be off antihistamines at least 4 days before study procedures.

${ }^{\dagger} P<.001$ comparing baseline eNO levels and eosinophil numbers per milligram in sputum (HVs vs AA patients). 


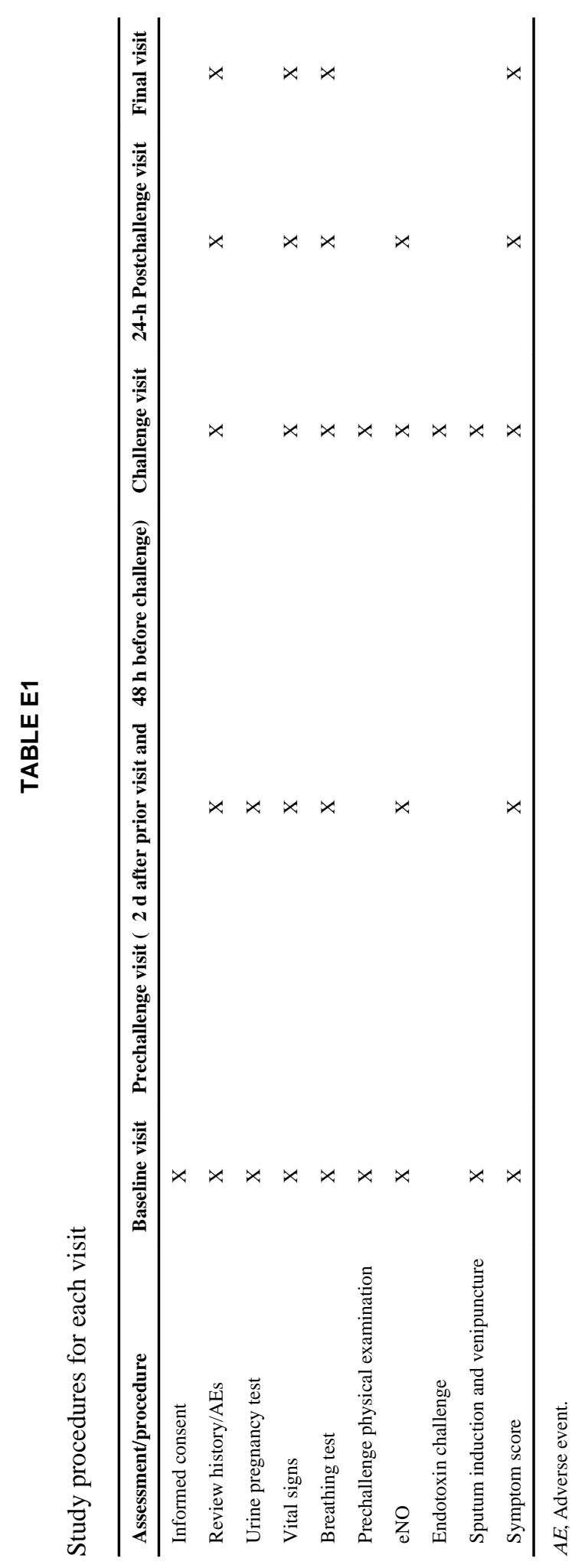

J Allergy Clin Immunol. Author manuscript; available in PMC 2013 October 01. 